\title{
Fibre structure of decametric type II radio bursts as a manifestation of emission propagation effects in a disturbed near-solar plasma
}

\author{
A. N. Afanasiev \\ ESTEC/ESA Noordwijk, The Netherlands \\ Institute of Solar-Terrestrial Physics, Irkutsk, Russia \\ Received: 19 June 2009 - Revised: 17 September 2009 - Accepted: 23 September 2009 - Published: 16 October 2009
}

\begin{abstract}
This paper addresses the fine structure of solar decametric type II radio bursts in the form of drifting narrowband fibres on the dynamic spectrum. Observations show that this structure appears in those events where there is a coronal mass ejection (CME) traveling in the near-solar space ahead of the shock wave responsible for the radio burst. The diversity in observed morphology of fibres and values of their parameters implies that the fibres may be caused by different formation mechanisms. The burst emission propagates through extremely inhomogeneous plasma of the CME, so one possible mechanism can be related to radio propagation effects. I suggest that the fibres in some events represent traces of radio emission caustics, which are formed due to regular refraction of radio waves on the large-scale inhomogeneous structure of the CME front. To support this hypothesis, I have modeled the propagation of radio waves through inhomogeneous plasma of the CME, taking into consideration the presence of electron density fluctuations in it. The calculations, which are based on the Monte Carlo technique, indicate that, in particular, the emission of the fibres should be harmonic. Moreover, the mechanism under consideration suggests that in solar observations from two different points in space, the observed sets of fibres can be shifted in frequency with respect to one another or can have a different structure. This potentially can be used for identifying fibres caused by the propagation effects.
\end{abstract}

Keywords. Radio science (Radio wave propagation) - Solar physics, astrophysics, and astronomy (Flares and mass ejections; Radio emissions)

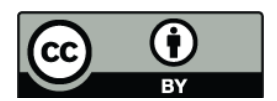

Correspondence to: A. N. Afanasiev (aafanasiev@rssd.esa.int)

\section{Introduction}

Solar radio observations show that dynamic spectra of type II bursts in the decametric and hectometric wavelength ranges quite often have fine structure in the form of irregular narrowband emissions. In some events, the fine structure is related to the interaction of two coronal mass ejections (CMEs) (Gopalswamy et al., 2001). In some cases, the burst fine structure has the form of narrowband drifting fibres (Fig. 1). The analysis of observational data has revealed that in these cases there was a CME on the propagation path of the shock wave generating the burst (ahead of the shock) (Chernov et al., 2007a, b). In some LASCO SOHO coronal images corresponding to such events, narrow elongated structures located behind the apparent leading edge of the CME can be seen. Chernov et al. (2007 a, b) suggest that these narrow structures (they refer to them as streamers or jets) are located in the CME tail and associate the appearance of the fibres with the passage of the shock wave through these jets. The fibres are hypothesized to represent enhanced resonance transition emission from the jets, caused by accelerated particles in the inhomogeneous plasma. Thus, it is assumed that the formed fine structure is due to properties of the emission source.

On the other hand, the process of radio waves propagation from the source to the point of observation can give rise to features in observed dynamic spectra. For instance, Wild and Roberts (1956) reported about an observation of strips in dynamic spectra of emission from a distant cosmic radio source. They associated the appearance of stripes with focusing and interference of the emission due to inhomogeneities in the Earth's ionosphere. Another example is a fine structure in the form of "nested arches", observed in dynamic spectra of Jovian decametric radio emission. This fine structure is likely to represent the combined effect of focusing and diffraction of the radio emission by some plasma formation in Jupiter's magnetosphere (Lecacheux et al., 1981).

Published by Copernicus Publications on behalf of the European Geosciences Union. 


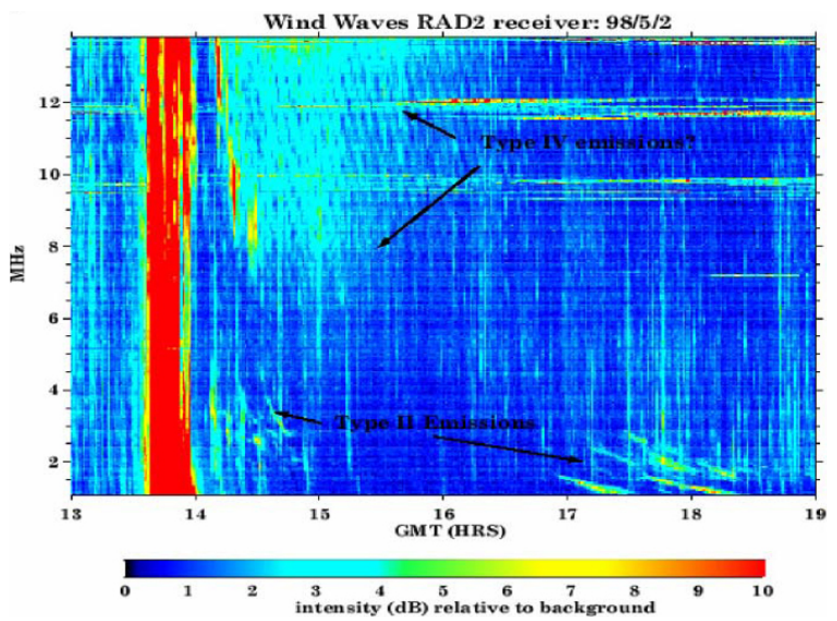

Fig. 1. Dynamic spectrum of type II burst with fine structure in the form of drifting narrowband fibres.

In the case under consideration, when a shock wave propagates in the wake of a CME, the emission generated at the shock front passes through CME's regions with increased electron density. In the case of a three-component CME, the electron density in the CME frontal structure exceeds the density of ambient plasma, on average, by an order of magnitude (Thernisien et al., 2006). Under such conditions, if one assumes that the generation of the radio emission occurs at the fundamental frequency or harmonic then even relatively small variations of the electron density in the CME frontal structure can give rise to significant refraction of radio waves and caustic focusing. Such density variations in the CME front could be related to the flux rope structure of the CME, if plasma concentrates in filamentary magnetic loops constituting the flux rope.

It should be noted that the formation of radio emission caustics has been studied in the context of various plasma media: in particular, as mentioned above, in the magnetosphere of Jupiter (Lecacheux et al., 1981) and in interstellar plasma (Melrose and Watson, 2006; Watson and Melrose, 2006). These investigations are based on the deterministic phase screen model (the latter paper considers a particular realization of a random phase screen), i.e. a problem of radio emission diffraction on a deterministic lens-like structure is considered. But for all that, the effect of electron density fluctuations lies beyond the bounds of examination. However, the scattering by density fluctuations results in smearing deterministic (regular) effects; in particular, it gives decrease in emission intensity on the regular caustics. Solar radio burst studies show that scattering effects play a significant role in the background (undisturbed) near-solar plasma. Moreover, solar radio sounding experiments from spacecraft imply (e.g. Bird et al., 1985; Woo and Armstrong, 1992) that the CME frontal structure and core have an enhanced level of turbulence (according to the relation $\left\langle\Delta N^{2}\right\rangle^{1 / 2} \sim N_{r}$, where
$N_{r}$ is the mean electron density of plasma, $\Delta N$ stands for the density fluctuations). Therefore, the scattering effects should be taken into consideration when studying the formation of caustics in the near-solar plasma.

The purpose of this paper is to investigate the possibility that the fibres of type II bursts can form due to radio propagation effects, taking into account both focusing properties of the CME and scattering properties of the near-solar plasma. For that, I consider the problem in quite general formulation without referring to any particular event and perform Monte Carlo modeling of the radio propagation near the Sun. An additional purpose of this study is to find some characteristic properties of the phenomena under consideration that could serve as a signature of the formation mechanism.

In Sect. 2, I state the idea of the fibre formation mechanism in a regular near-solar plasma. In Sect. 3, I give some details of the Monte Carlo method as applied to the solar radio emission. I describe models for a CME and random irregularities, which were used in calculations, in Sect. 4. In Sect. 5, I present results of calculations and their discussion and conclude in Sect. 6.

\section{Mechanism for the fibre formation}

I suggest that the fibers in type II dynamic spectra can be formed due to the fact that the radio emission generated at the shock front propagating in the wake of the CME encounters CME-associated regions with enhanced electron density. Of particular attention is the expanded frontal structure, which has inhomogeneous internal structure. Ray-tracing calculations for a simple regular model of the near-solar plasma (which specifies large-scale variations in the electron density distribution only and does not take into account any fluctuations) have shown that a region of enhanced density associated with the CME frontal structure can give rise to ray caustics, if there are some variations in density in such a region. Figure 2 presents the corresponding ray patterns for an arbitrary point source.

Type II radio emission is known to be generated at the shock front at fundamental and/or harmonic frequencies which, in the simplest case of a spherically symmetric coronal model, are functions of the source height. Therefore, if we assume that the emission is generated by point sources distributed throughout the shock front, then each such a source will emit at some particular frequency (fundamental and/or harmonic). Furthermore, each such a source will produce its own caustics which have different positions in space. Some caustics produced by different sources (correspondingly at different frequencies) can come directly into the view of the observer and, hence, the observer will record an increase in intensity at these frequencies. The frequencies of focusings change with time because of the outward movement of the shock wave and CME, which produces the traces of fibres on the dynamic spectrum. 

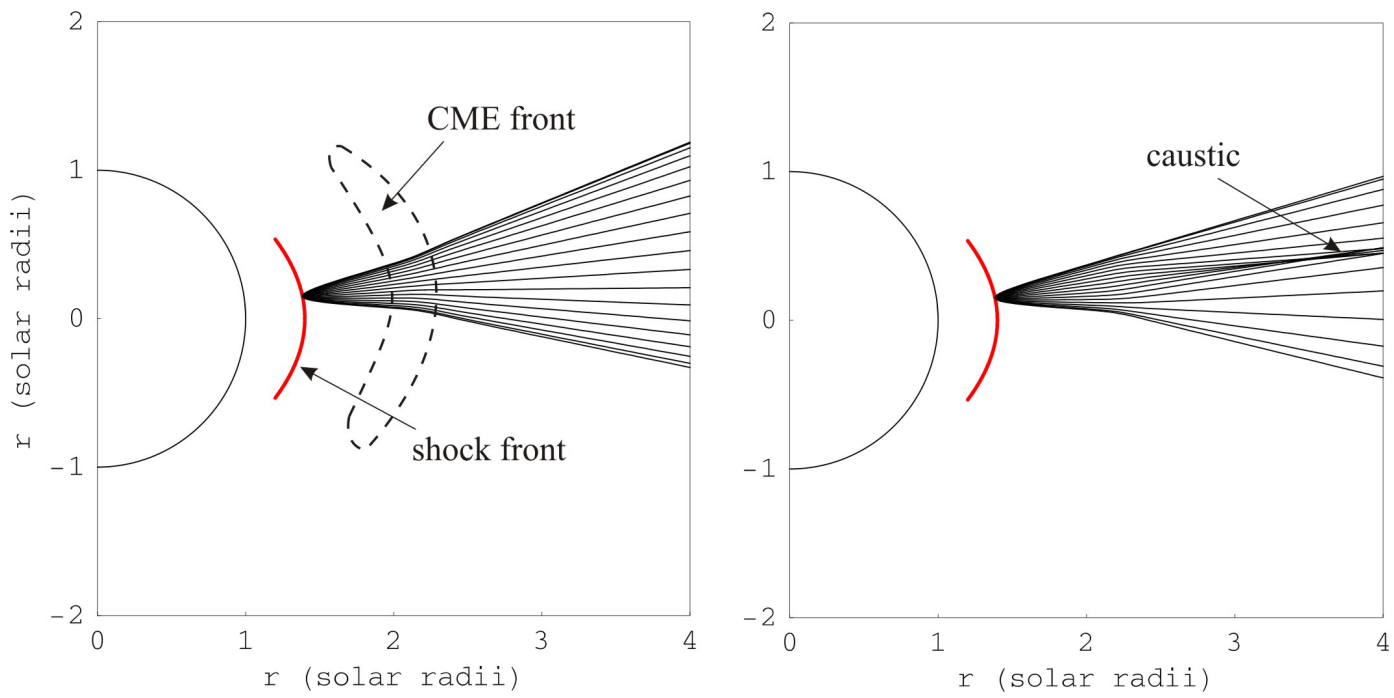

Fig. 2. Characteristic ray trajectory patterns for an individual point source at the shock front. On the left panel, the electron density in the CME frontal structure gradually falls from its central part towards the edges. Therefore, only a broadening of the ray cone occurs. On the right panel, there is a local region with decreased electron density in the CME front, which produces a caustic.

\section{Scattering}

Apart from the regular refraction, the other crucial factor for the propagation of radio emission in the near-solar plasma is its scattering by electron density irregularities. The scattering causes smearing of the caustics and thus reduces the magnitude of focusing. Therefore, it is of importance to take the scattering into consideration. One way to do this is to use the geometrical optics (GO) method, which, as is known, works well in those cases where the ambient plasma is significantly inhomogeneous and the effects of regular refraction are important. I apply the Monte Carlo technique, which is based on the GO method. This numerical approach gives one an opportunity to take into account more fully the scattering of radio waves on density irregularities (to include the large-angle scattering in addition to the small-angle refractive scattering described by the geometrical optics) and their refraction in a strongly inhomogeneous medium, i.e. to study the influence of scattering on the emission focusing.

The Monte Carlo method has been used for many years to study the scattering of solar radio emissions by density fluctuations (Fokker, 1965; Steinberg et al., 1971; Steinberg, 1972; Riddle, 1974; Thejappa et al., 2007). Details about the method and conditions of its applicability can be found in Steinberg et al. (1971) for the case of a Gaussian power spectrum of density irregularities and in Thejappa et al. (2007) for a power-law (Kolmogorov) spectrum. However, it is worth indicating some key points of the method, which are necessary in what follows.

The Monte Carlo method approximates the scattering as irregular refraction of rays. In other words, it deals with tracing rays step by step. At each step $\Delta S$, it is necessary to solve the ray-tracing equations, which can be written in the following form (Kravtsov and Orlov, 1990):

$$
\begin{aligned}
& \frac{d \boldsymbol{R}}{d s}=\frac{\boldsymbol{T}}{\mu}, \\
& \frac{d \boldsymbol{T}}{d s}=\nabla \mu,
\end{aligned}
$$

where $\mu=\mu(\boldsymbol{r})$ is the refractive index which is a function of coordinates, $s$ is the ray path length,

$\boldsymbol{R} \equiv\left(\begin{array}{c}x \\ y \\ z\end{array}\right) \quad$ and $\quad \boldsymbol{T} \equiv\left(\begin{array}{c}T_{x} \\ T_{y} \\ T_{z}\end{array}\right)$

are the position and direction cosine vectors of the ray, respectively, and

$T_{x}^{2}+T_{y}^{2}+T_{z}^{2}=\mu^{2}$.

The step $\Delta S$ is chosen such that the approximations of smallangle scattering and quasi-linearity of rays (see, e.g. Steinberg et al., 1971) are valid. At the end of each step, a random vector is added to the direction of the ray, the components of which are taken from a Gaussian distribution with zero mean and the variance which for a Kolmogorov power law spectrum of irregularities has the form (Thejappa et al., 2007):

$$
\left\langle\Psi^{2}\right\rangle_{K}=\pi \frac{f_{p}^{4}}{f^{4}} \frac{\varepsilon^{2}}{\mu^{4} l_{i}^{1 / 3} l_{o}^{2 / 3}} \Delta S,
$$

where $f$ is the emission frequency, $f_{p}(r) \approx 9 \times 10^{-3} \sqrt{N_{r}(r)}$ is the plasma frequency in $\mathrm{MHz}$ in a regular medium, which varies directly with the regular electron density $N_{r}(r) ; \varepsilon$ 
is called the relative level of density fluctuations; $l_{i}(r)$ and $l_{o}(r)$ are the inner and outer scales of plasma turbulence, respectively.

Earlier, the similar calculations of the scattering in the solar corona were conducted based on the assumption that the power spectrum of density fluctuations is a Gaussian. In this case, the formula for the characteristic scattering angle has the following form (Steinberg et al., 1971):

$$
\left\langle\Psi^{2}\right\rangle_{G}=\frac{\sqrt{\pi}}{2} \frac{f_{p}^{4}}{f^{4}} \frac{\varepsilon^{2}}{\mu^{4} l} \Delta S
$$

where $l$ is the characteristic scale of density inhomogeneities. It is evident that

$$
\left\langle\Psi^{2}\right\rangle_{K}=\left\langle\Psi^{2}\right\rangle_{G} \text {, if } \quad l(\boldsymbol{r})=\frac{l_{i}^{1 / 3}(\boldsymbol{r}) \cdot l_{o}^{2 / 3}(\boldsymbol{r})}{2 \sqrt{\pi}} .
$$

Since at heliocentric distances $r<10 R_{S}\left(R_{S}\right.$ is the solar radius), which are of interest in this study, the power spectrum of density fluctuations inside the CME is very close to Kolmogorov (see Sect. 4), one should use Eq. (3) when modeling the scattering effects. However, the condition (5) allows us to apply Eq. (4), which is easier to do in the situation where the relations for $l_{i}(\boldsymbol{r})$ and $l_{o}(\boldsymbol{r})$ are not clear (e.g. in the wake of the CME). The formulas for $l_{i}(\boldsymbol{r})$ and $l_{o}(\boldsymbol{r})$ valid in the background medium can be used as a reference.

After tracing a large number of rays from the source to the Earth's orbit, the traditional way is to divide the orbit into small sectors and to count the number of rays arrived at each particular sector (the size of such a sector is usually characterized by the corresponding central angle $\Delta \psi$ subtending the sector in the Sun's center). The inferred distribution of scattered rays on the Earth's orbit contains information about the directivity of the source.

I have found that in order to study how the scattering affects regular caustics in inhomogeneous plasma, it is necessary to trace many more rays as compared with the case of a spherically symmetric model. Therefore, I have confined myself to considering a two-dimensional case.

\section{The models used}

The electron density distribution in the near-solar plasma can be divided into two components:

$$
N_{e}(\boldsymbol{r})=N_{r}(\boldsymbol{r})+\Delta N(\boldsymbol{r}),
$$

where the function $N_{r}(\boldsymbol{r})=N_{b}(r)+N_{\mathrm{CME}}(\boldsymbol{r})$ represents a regular component composed of a spherically symmetric background density distribution $N_{b}(r)$ and a function $N_{\mathrm{CME}}(\boldsymbol{r})$ specifying the mean density distribution in the CME; the function $\Delta N(\boldsymbol{r})$ describes electron density fluctuations in the near-solar plasma. It is assumed that $\left\langle\Delta N^{2}\right\rangle^{1 / 2} \ll N_{b}(\boldsymbol{r})$, and $\left\langle\Delta N^{2}\right\rangle^{1 / 2} \ll N_{\mathrm{CME}}(\boldsymbol{r})$.

For the background density distribution $N_{b}(r)$, I adopt the functional form proposed by Leblanc et al. (1998), which was also used for estimations by Chernov et al. (2007a, b). As far as the density distribution in the CME is concerned, I rely on models used for reproducing its large scale structure. In this kind of models, the CME represents just a shell with enhanced electron density (e.g. Thernisien et al., 2006). In other words, such models reproduce only the frontal structure of the CME and neglect other features such as the cavity and core. I consider a two-dimensional case and specify the model of the CME frontal structure in the form of a semicircular plasma layer. In the heliocentric Cartesian reference frame, it can be written in the following form:

$$
\begin{aligned}
& N_{\mathrm{CME}}(x, y)=Q N_{b}(H) K(x, y) \\
& \quad \exp \left\{-\frac{\left(\sqrt{(y-H+R)^{2}+x^{2}}-R\right)^{2}}{\sigma^{2}}\right\} \\
& R(H)=\left(1-\frac{1-\kappa}{1+\sin \gamma}\right) H, \\
& K(x, y)=1+\delta Q \cos ^{2}\left[\frac{2 \pi}{\varphi_{l}} \arctan \left(\frac{x}{y}\right)\right],
\end{aligned}
$$

where $H$ is the height (relative to the Sun's center) of the maximum of the plasma layer, $\sigma$ characterizes the layer thickness, $Q$ defines the mean value of electron density in the layer. The function $K(y)$ represents the intrinsic inhomogeneous structure of the layer, which may result from the flux-rope structure of the CME. The parameter $\varphi_{l}$ specifies the angular size of the electron density variation and $\delta$ characterizes the magnitude of the variation (Fig. 3). The expression for $R(H)$ is obtained in terms of the CME model used in calculations reported by Thernisien et al. (2006). The constants $\kappa$ and $\gamma$ in the model suggested by Thernisien et al. (2006) characterize the aspect ratio and the angular width, respectively. Since it is necessary to specify the characteristic geometrical scales of the CME, the typical values of these parameters: $\kappa=0.43$, and $\gamma=25^{\circ}$ are used.

As far as the electron density fluctuations inside the CME are concerned, their properties are not known in detail. The radio sounding experiments using signals from spacecraft have shown that the slope of the turbulence power spectrum increases significantly behind the CME front (Woo and Armstrong, 1992; Efimov et al., 2008). Specifically, according to Woo and Armstrong (1992), the power exponent $p$ of the turbulence spectrum at small heliocentric distances $\left(r<10 R_{S}\right)$ changes from $p \approx 3.0$ in the region before the leading edge to $p \approx 11 / 3$ after the leading edge. However, the values of parameters $l_{i}, l_{o}$ and $\varepsilon$ inside the CME are not clear.

As regards the outer scale of turbulence, $l_{o}$, I consider the following well known relation (see, Efimov et al., 2002; Thejappa et al., 2007):

$l_{o}(r)=8.82 \times 10^{-2} r^{0.82}[\mathrm{AU}]$,

where $r$ is in units of AU. As for the inner scale, $l_{i}$, it is known that it increases with the heliocentric distance (Coles 

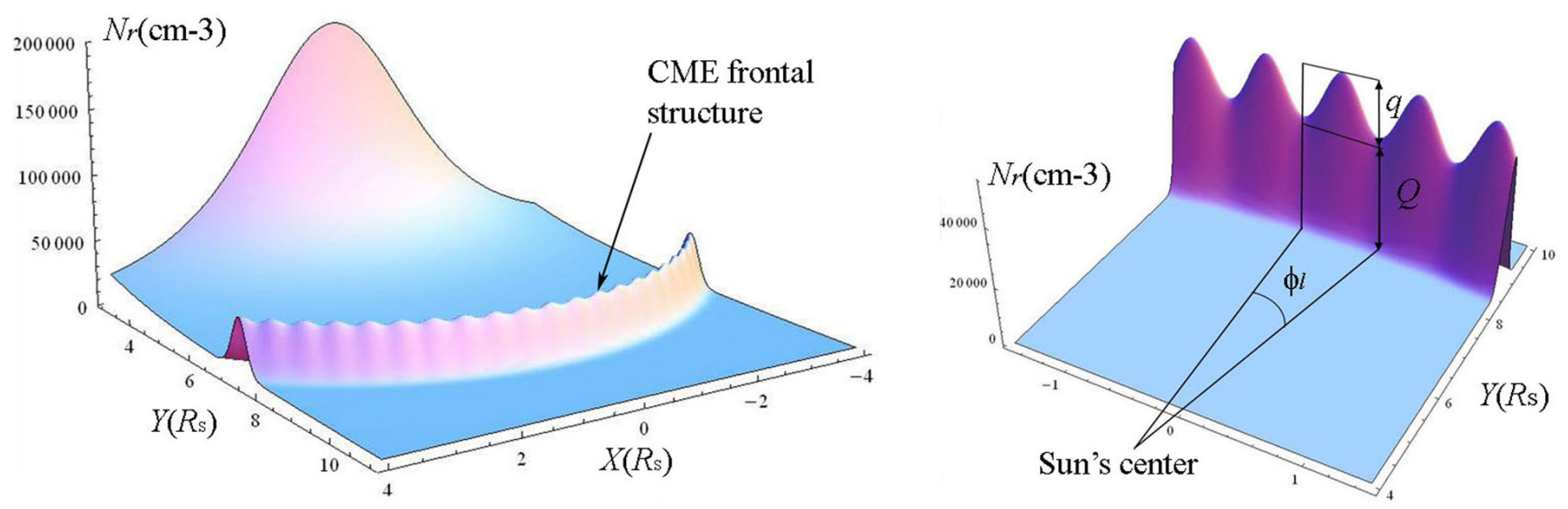

Fig. 3. Left panel: The regular electron density distribution $N_{e}(r)$ in the near-solar plasma. Right panel: Density variation parameters, $\varphi_{l}$ and $\delta=q / Q$ in the CME front.

and Harmon, 1989) from about $1 \mathrm{~km}$ at $r \approx 3 R_{S}$ to $50 \mathrm{~km}$ at $r \approx 25 R_{S}$.

To calculate the scattering, I used Eq. (4), supposing $l=$ const everywhere in space, and chose values of $l$ around $l^{*}=\frac{1}{2 \sqrt{\pi}} l_{i}^{1 / 3}\left(r_{0}\right) l_{o}^{2 / 3}\left(r_{0}\right)$ (here $r_{0}$ is the heliocentric distance of the source).

In many papers dealing with the modeling of radio emission scattering, the parameter $\varepsilon=\left\langle\Delta N^{2}\right\rangle^{1 / 2} / N_{r}$ is assumed to be constant throughout the medium (e.g. Bastian, 1994). That was observationally supported by Woo (1978) down to $r \approx 1.7 R_{S}$. Bavassano and Bruno (1995) deduced from the in-situ measurements onboard the HELIOS spacecraft that $\varepsilon \approx 6 \ldots 10 \%$ at the distance of about $65 R_{S}$ from the Sun. On the other hand, based on the analysis of radio sounding observations with the VENERA-15, -16 spacecraft, Rubtsov et al. (1987) obtained $\varepsilon=3 \ldots 5 \%$ at $r \approx 5.5 R_{S}$. They also found that $\varepsilon$ decreased with the approach to the Sun. The mentioned results indicate that it is reasonable to take $\varepsilon=3 \ldots 10 \%$ for the calculations.

\section{Results and discussion}

To study the effect of scattering on the regular focusing, I calculated distributions of scattered rays on the Earth's orbit. The rays are assumed to be emitted by point sources distributed throughout the shock front (correspondingly, at different frequencies). The calculations have been performed for different relative positions of the shock wave and CME. An example of such distributions for the geometry depicted in Fig. 4 is shown in Fig. 5. The distributions presented suit a point source located at the apex of the shock front. For the purposes of this study, it is enough to consider just a small cone of rays leaving the source in the forward direction (see Fig. 4). This allows one to reduce the number of rays to be traced. To obtain the histograms in Fig. 5, 2000 rays uni-

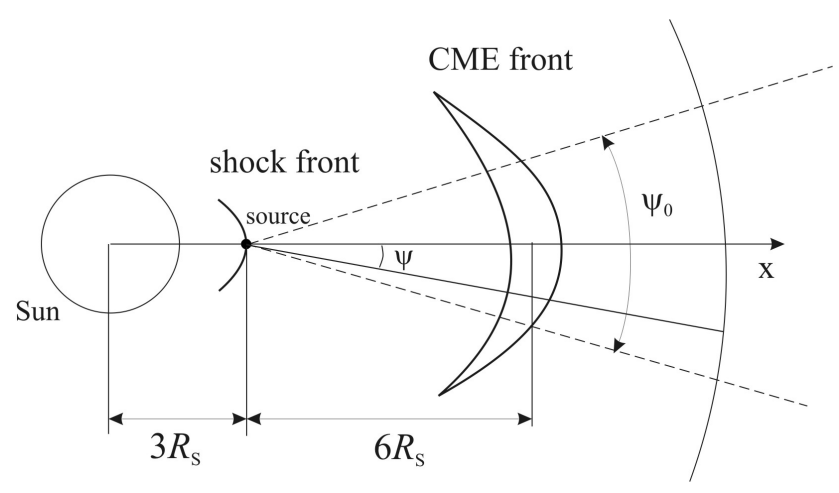

Fig. 4. Geometry adopted for the calculations.

formly distributed within a half of the two-dimensional initial cone with $\psi_{0}=40^{\circ}$ were traced. The averaging interval for the scattered rays is $\Delta \psi=0.25^{\circ}$.

It is evident from Eq. (4) that the scattering is most sensitive to the "level" of inhomogeneity of plasma $\varepsilon$ and the ratio $f_{p} / f$. The histograms in the left column of Fig. 5 have been obtained under the assumption that the source emits at the fundamental frequency $f=1.05 f_{p}$ and those in the right column correspond to the assumption of the harmonic source. The upper histograms $(\varepsilon=0)$ correspond to the case of a regular medium. The peaks in the distributions represent the effect of radio emission focusing on the caustics. It can be seen that more intensive density fluctuations produce stronger smearing of the caustics. However, the focusing of the harmonic emission remains to be clearly seen for the values of $\varepsilon$ consistent with those obtained from the observations. It is also clear from the histograms that the scattering significantly reduces the focusing on the caustics so that it is unlikely that the intensity of fibres produced by the suggested mechanism can exceed the background more than a few times. 

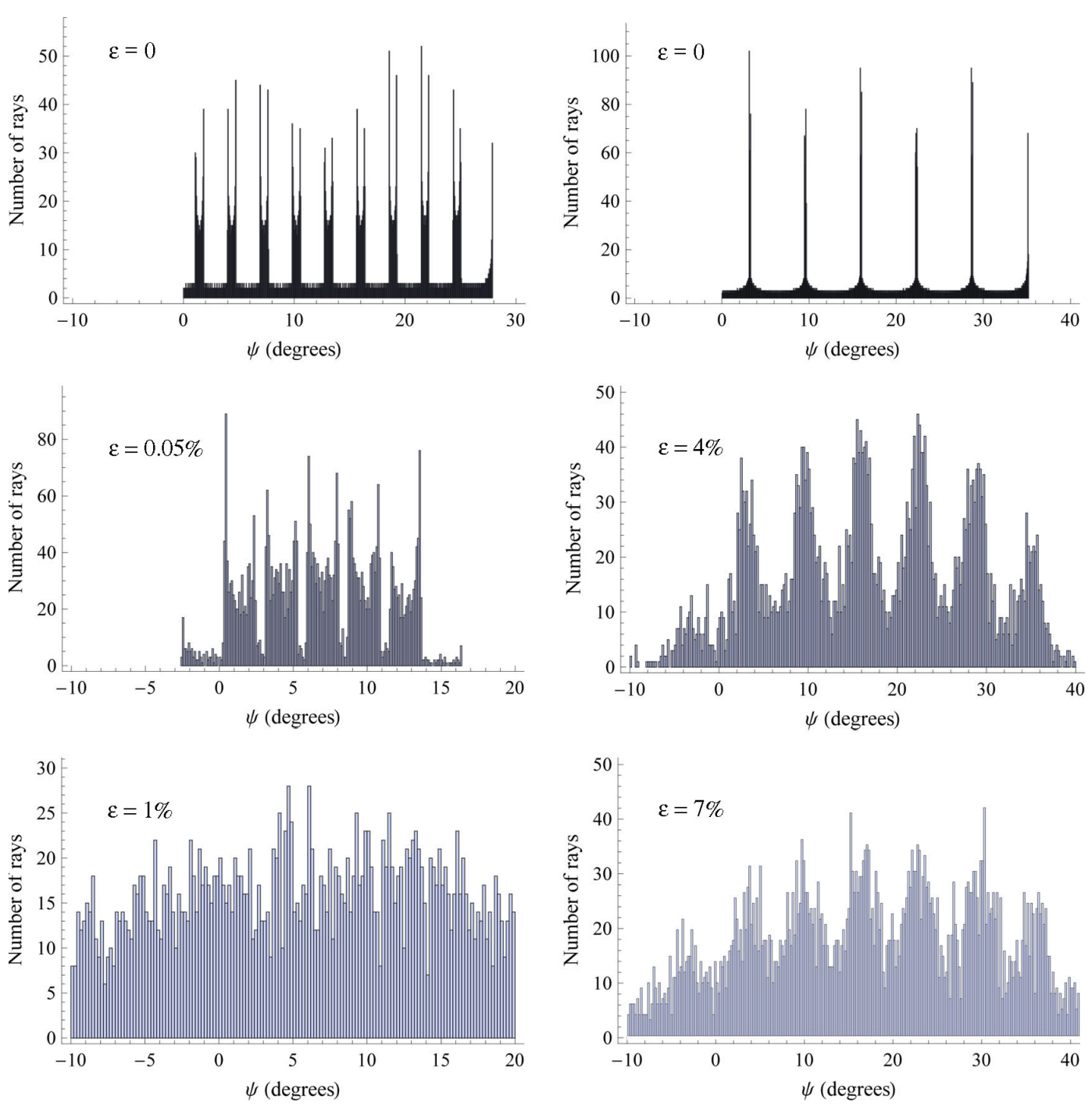

Fig. 5. Distributions of scattered rays on the Earth's orbit for the geometry in Fig. 4 for different values of turbulence level $\varepsilon$. The left column of histograms corresponds to the fundamental emission $\left(f=1.05 f_{p}, f_{p} \approx 4 \mathrm{MHz}\right)$, the right column - to the harmonic emission $\left(f=2 f_{p}\right)$. The values of the turbulence parameters: $l_{i}=3 \mathrm{~km}, l_{o}=4.1 \times 10^{5} \mathrm{~km}, l^{*} \approx 2.3 \times 10^{3} \mathrm{~km}$, and $l=2.2 l^{*}$. The values of the CME regular structure parameters: $Q=7.5 ; \varphi_{l}=2^{\circ}, \delta=7 \%$ (left column); $\varphi_{l}=4.3^{\circ}, \delta=50 \%$ (right column).

The calculations have also revealed that the formation of the caustic focusing depends not only on the parameters of random irregularities but also on the regular structure of the CME front. If the produced caustics lie close to each other on the Earth's orbit, then quite weak irregularities smear the caustic structure entirely. Thus, the fibres potentially contain information about parameters (the characteristic size and gradient) of density variations in the frontal structure of the CME.

Figure 6 shows two distributions of scattered rays emitted by two sources located on a parabolic-shaped shock front, which is specified by the relation $y=k x^{2}$ with $k=0.4$. For the calculation, the geometry presented in Fig. 4 was adopted. One of the sources is located at the apex of the front and the other one - on the branch of the parabola. One can see the shift of the distributions with respect to one another, which implies that the position (or structure) of sets of the "caustic" fibres on the dynamic spectrum observed from two different points on the Earth's orbit will be different. Therefore, measurements of the dynamic spectra of fibres (or other types of fine structure) at different points in space can be used for identifying those features that are caused by the propagation effects.

Note that the density variations along the leading edge of the CME may not be uniform in magnitude and spacing. In this case, the distribution of caustic features (produced by one source on the shock front) along the Earth's orbit should be nonuniform. Therefore, the frequency separation between 


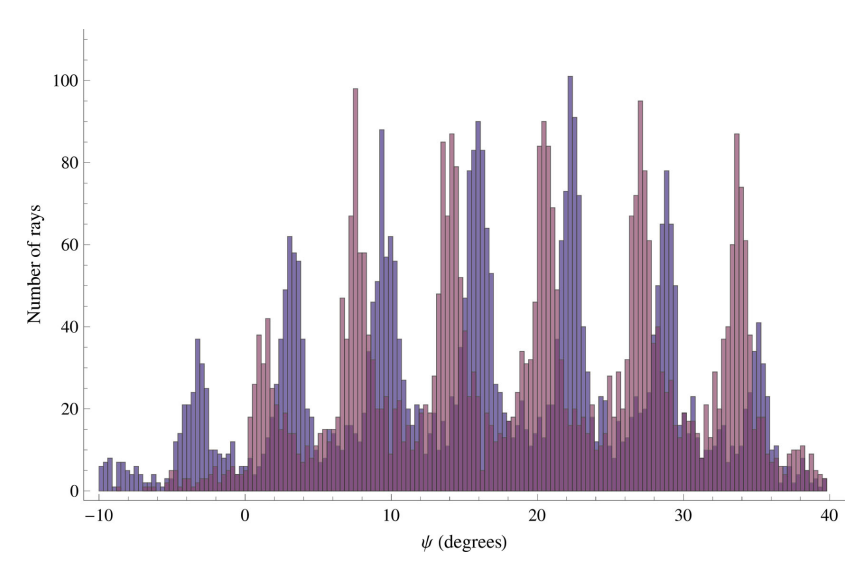

Fig. 6. Relative shift of two distributions of scattered rays from two different point sources at the shock front.

the adjacent fibres in the dynamic spectrum may be different for different pairs of fibres, which is actually observed. Besides, it is more likely, in this case, to expect a different structure of sets of fibres (for example, different number of fibres) observed in two different points in space than just a relative shift in frequency of the sets.

It should also be noted that in three-dimensional space the caustics represent surfaces but not curves as in the twodimensional problem. The structure of such caustic surfaces may be complicated, depending on the structure of the density variations in the CME. Therefore, either some decrease or increase in the radio wave intensity on the caustics as compared with the two-dimensional case may be possible.

\section{Conclusions}

The results of this study support the hypothesis that the fine structure of type II solar radio bursts in the form of drifting narrowband fibres can be the result of focusing of the radio emission due to the inhomogeneous structure of the CME (probably, of its frontal structure). The calculations of the radio wave scattering based on the Monte Carlo technique have shown the following.

1. The scattering significantly reduces the magnitude of focusing so that the fundamental emission becomes totally smeared for reliable values of the plasma turbulence parameters. However, the harmonic emission focusing remains to be well pronounced in the source directivity patterns for the values of the turbulence parameters consistent with those obtained from the observations. Therefore, the emission of the "caustic" fibres should be harmonic.

2. It is unlikely that the intensity of the fibres produced by the suggested mechanism can exceed the background more than a few times.
3. The "caustic" fibres potentially contain information about values of the turbulence parameters inside CMEs and about the structure of the CME front.

4. In solar observations from two different points in space, the observed sets of "caustic" fibres can be shifted in frequency with respect to one another or can have different structure. This potentially can be used for identifying fibres caused by the propagation effects.

Acknowledgements. The author thanks Bernhard Fleck and Daniel Mueller for continuing support and advice. $\mathrm{He}$ also thanks Michael Kaiser, Mike Hapgood, Andreas Klassen and Jasmina Magdalenic for detailed discussion of this work at the Workshop.

Topical Editor R. Forsyth thanks R. MacDowall and another anonymous referee for their help in evaluating this paper.

\section{References}

Bastian, T. S.: Angular scattering of solar radio emission by coronal turbulence, Astrophys. J., 426, 774-781, 1994.

Bavassano, B. and Bruno, R.: Density fluctuations and turbulent Mach numbers in the inner solar wind, J. Geophys. Res., 100, 9475-9480, 1995.

Bird, M. K., Volland, H., Howard, R. A. Koomen, M. J., Michels, D. J., Sheeley, N. R., Amstrong, J. W., Seidel, B. L., Stelzried, C. T., and Woo, R.: White-light and radio sounding observations of coronal transients, Solar Phys., 98, 341-368, 1985.

Chernov, G. P., Kaiser, M. L., Bougeret, J.-L., Fomichev, V. V., and Gorgutsa, R. V.: Fine structure of solar radio bursts observed at decametric and hectometric waves, Solar Phys., 241, 145-169, 2007a.

Chernov, G. P., Stanislavsky, A. A., Konovalenko, A. A., Abranin, E. P., Dorovsky, V. V., and Rucker, G. O.: Fine structure of decametric type II radio bursts, Astron. Lett., 33, 192-202, $2007 \mathrm{~b}$.

Coles, W. A. and Harmon, J. K.: Propagation observations of the solar wind near the Sun, Astrophys. J., 337, 1023-1034, 1989.

Efimov, A. I., Chashei, I. V., Samoznaev, L. N., Andreev, V. E., Bird, M. K., Edenhofer, P., Plettemeier, D., and Wohlmuth, R.: The outer scale of solar-wind turbulence from GALILEO coronal-sounding data, Astron. Rep., 46, 579-590, 2002.

Efimov, A. I., Rudash, V. K., Samoznaev, L. N., Bird, M. K., Chashei, I. V., and Plettemeier, D.: Coronal radio-sounding detection of a CME during the 1997 Galileo solar conjunction, Adv. Space Res., 42, 110-116, 2008.

Fokker, A. D.: Coronal scattering of radiation from solar radio sources, Bull. Astron. Inst. Netherlands, 18, 111, 1965.

Gopalswamy, N., Yashiro, S., Kaiser, M. L., Howard, R. A., and Bougeret, J.-L.: Radio signatures of coronal mass ejection interaction: Coronal mass ejection cannibalism?, Astrophys. J., 548, L91-L94, 2001.

Kravtsov, Yu. A. and Orlov, Yu. I.: Geometrical Optics of Inhomogeneous Media, Springer-Verlag, Berlin Heidelberg New York, 1990.

Leblanc, Y., Dulk, G. A., and Bougeret, J.-L.: Tracing the electron density from the corona to $1 \mathrm{AU}$, Solar Phys., 183, 165-180, 1998. 
Lecacheux, A., Meyer-Vernet, N., and Daigne, G.: Jupiter's Decametric Radio Emission: A nice problem of optics, Astron. Astrophys., 94, L9-L12, 1981.

Melrose, D. B. and Watson, P. G.: Scintillation of radio sources: The role of caustics, Astrophys. J., 647, 1131-1141, 2006.

Riddle A. C.: On the observation of scattered radio emission from sources in the solar corona, Solar Phys., 35, 153-169, 1974.

Rubtsov, S. N., Yakovlev, O. I., and Efimov, A. I.: Plasma density inhomogeneity and solar wind kinetic energy as deduced from radio sounding data using the "VENERA-15 and -16" spacecraft, Kosmicheskiye Issledovaniya, 25, 620-625, 1987.

Steinberg, J.-L.: Coronal scattering at hectometer and kilometer wavelengths, Astron. Astrophys., 18, 382-389, 1972.

Steinberg, J.-L., Aubier-Giraud, M., Leblanc, Y., and Boischot, A.: Coronal scattering, absorption and refraction of solar radio bursts, Astron. Astrophys., 10, 362-376, 1971.
Thejappa, G., MacDowall, R. J., and Kaiser, M. L.: Monte Carlo simulation of directivity of interplanetary radio bursts, Astrophys. J., 671, 894-906, 2007.

Thernisien, A. F. R., Howard, R. A., and Vourlidas, A.: Modeling of flux rope coronal mass ejections, Astrophys. J., 652, 763-773, 2006.

Watson, P. G. and Melrose, D. B.: Scintillation of radio sources: The signature of a caustic, Astrophys. J., 647, 1142-1150, 2006.

Wild, J. P. and Roberts, J. A.: The spectrum of radio-star scintillations and the nature of irregularities in the ionosphere, J. Atmos. Terr. Phys., 8, 55-75, 1956.

Woo, R.: Radial dependence of solar wind properties deduced from HELIOS $1 / 2$ and Pioneer 10/11 radio scattering observations, Astrophys. J., 219, 727-739, 1978.

Woo, R. and Armstrong, J. W.: Observations of the electron density spectrum near the Sun during spectral broadening transients, in: Solar Wind Seven; Proceedings of the 3rd COSPAR Colloquium, Goslar, Germany, 16-20 September 1991, 583-586, 1992. 